\title{
A Criteria Catalog Based Methodology for Analyzing Service Management Processes
}

\author{
Michael Brenner ${ }^{1}$, Igor Radisic ${ }^{1}$, and Martina Schollmeyer ${ }^{2}$ \\ 1 University of Munich, Munich Network Management Team *, Dep. of Informatics, \\ D-80538 Munich, Germany \{brenner|radisic\} @informatik. uni-muenchen. de \\ 2 BMW Group, User Service Center, D-80788 Munich, Germany \\ martina.schollmeyer@bmw.de
}

\begin{abstract}
To achieve cost-efficient and effective provisioning of IT Services, a business process oriented IT service management is needed. Before they can be managed though, existing processes have to be analyzed.

This paper proposes the usage of criteria catalogs for this task. Using a collection of "best practices", process characteristics of optimal processes can be identified, leading to an assemblage of criteria for high process quality. The methodology presented in this paper allows the organization of these criteria into a catalog and subsequent computation of a numerical quality rating.

As a proof-of-concept application, a generic catalog for analyzing Incident Management processes, using the IT Infrastructure Library (ITIL) as a best practices database, is presented, and the experiences regarding its application for evaluating the User Service Center of the BMW Group are described.
\end{abstract}

\section{Introduction}

IT has become a critical factor for corporate success, not only for E-Business and ECommerce companies, but for companies of all industrial sectors [3]. The often heavy investments needed for installing, operating and managing the IT infrastructure have become a serious issue, since IT service providers are facing tight constraints regarding human resources and budget. Therefore, improving the efficienc y of service provisioning and thereby maximizing the return-on-investment (ROI) is one of the most important tasks of service providers. This fact has initiated a shift from technology-oriented to more customer- and therefore service-oriented IT Management, which is often referred to as IT Service Management (ITSM) [1]. Consequently, following techniques applied in contemporary business management, ITSM is increasingly adopting a business process oriented approach to implement a cost-effective and efficient end-to-end (service) management solution.

Prior to optimizing existing service management processes, an in-depth analysis of the process implementation has to be done in order to identify quality decreasing or deficient parts. However, analysis techniques, such as benchmarking [6], developed so

\footnotetext{
* The authors wish to thank the members of the Munich Network Management (MNM) Team for valuable comments on previous versions of this paper. The MNM Team directed by Prof. Dr. H.-G. Hegering is a group of researchers of the University of Munich, the Munich University of Technology and the Leibniz Supercomputing Center.
} 
far imply an enormous effort with partly uncertain success regarding the usability of the achieved results. Hence, as this is usually a very time consuming and costly task, our goal is to develop an efficient and practicable technique for evaluating processes in the area of IT service management.

In this paper, we are presenting an easy-to-use, but powerful methodology for analyzing and evaluating service management processes. The basic idea is to develop a criteria catalog for quality measurement on the basis of a widely accepted framework for implementing service management processes, namely the IT Infrastructure Library (ITIL) recommendations [8]. The criteria catalog itself serves as a domain-specific (regarding the same type of process), but scenario-independent instrument for analyzing management processes, assigning numerical scores to various aspects of process quality, when being applied to a concrete scenario. Once developed, a criteria catalog can be applied multiple times in various scenarios for process evaluation purposes. This enormously reduces the effort usually required for process analysis. Additionally, as the overall achieved score is computed on basis of single criterion ratings, quality decreasing parts can be located easily.

The remainder of the paper is organized as follows: In Section 2 we discuss related work with a special focus on previously developed process analysis methods and the ITIL framework. Afterwards, in Section 3 the methodology for creating a criteria catalog is presented which is capable of measuring the quality of service management processes. Following the presented methodology, we are developing a criteria catalog for evaluating the Incident Management process in Section 4. Additionally, a snapshot of using the criteria catalog in a concrete scenario (i.e. the User Service Center of the BMW Group) is demonstrated. Section 5 concludes the paper and presents further work.

\section{Related Work}

For successful service management, a service provider needs to monitor and manage not only the technical infrastructure, but must organize the business processes of its operations in the best possible way. A process can be defined as "a connected series of actions, activities, changes etc. performed by agents with the intent of satisfying a purpose or achieving a goal" [8].

The analysis and redesign of business processes is commonly referred to as Business Process Reengineering (BPR) [2]. The framework that Kettinger et al. [5] derived from analyzing a multitude of BPR methodologies, defines six phases of a BPR project: Envision, Initiate, Diagnose, Redesign, Reconstruct and Evaluate. In the Diagnose phase, the existing process is documented and analyzed. However, a useful qualitative analysis of a process often proves all but impossible without comparing it to other process designs. Organizations are therefore often looking for ways of gaining knowledge about the design of similar processes from outside sources. Note that the analysis of a process is not to be confused with the quantitative measuring of its actual, real-life performance, which is part of the Evaluate phase.

Benchmarking [6] may be the most popular of the schemes for attaining and using external input for rating and improving processes. Benchmarking poses several challenges though. Especially finding a suitable benchmarking partner with a "best" design 
of similar processes who is willing to share this knowledge is often difficult. A different approach to ease the search for optimal process designs is the use of best practices collections [4]. These best practices databases are usually assembled by consultancies, that, due to the insights they have gained in advising on a multitude of benchmarking projects, are able to collect a large number of "best" processes across various industries. To our knowledge, there are no formal approaches to incorporating this kind of expertise into process analysis techniques, though.

The IT Infrastructure Library (ITIL) [8] is a public domain collection of best practices for a process-oriented IT service management. The ITIL defines ten core processes (as well as one business function) of ITSM and supplies guidelines and recommendations for implementing them. However, it describes its guidelines in a relatively loose structure and does not provide comprehensive formal models of its processes. Comparing an existing, documented process to these guidelines therefore cannot be accomplished in a straight-forward way.

The criteria catalog methodology, a body of methods and rules for creating and applying the tool of the same name, was devised by the MNM Team. The methodology, originally developed as a generic decision making tool, pursues a structured approach to evaluations, by identifying criteria that a perfect solution should meet, weighting them according to their importance and compiling them into a catalog. A criterion can either include a concise method for assigning "fulfillment values", or this value has to be derived from the evaluation of its sub criteria, that are representing various aspects of it.

\section{Methodology}

As aforementioned, we are not aware of any formal approaches of using best practices databases for analyzing the quality of processes. Thus, our goal is to provide an easy, efficient and, in particular, formalized procedure for analyzing the quality of ITSM processes, when using a best practices database. We therefore develop an uncomplicated and efficient tool, i.e. a criteria catalog, that aids in evaluating existing processes. This tool produces a numeric rating of process elements as well as of the whole process, by taking advantage of best practices knowledge during the Diagnose phase. On the basis of these ratings, shortcomings in the process implementation are revealed. Thus, identifying areas that need improvement is easily possible. In this section the methodology for both developing and applying this criteria catalog is presented. In Section 3.1 an overview over the developed methodology is provided, with a special emphasis on how to derive the criteria catalog. Note that in Section 4 this methodology is applied for a specific process illustrating many steps of the methodology. As the criteria catalog is the main instrument for carrying out the process analysis, a more detailed description of its main characteristics and general structure is given in Section 3.2 and Section 3.3, respectively.

\subsection{Analysis Methodology}

Basically the methodology, which is depicted in Fig. 1, is divided into two parts, a scenario-independent and a scenario-specific one: the first part deals with developing a 
domain-specific (regarding the process of interest), but reusable (regarding its application in different scenarios) criteria catalog, while in the second part the criteria catalog is applied to an existing process, whose quality we want to measure (and this is, of course, scenario-specific). Thus, once developed, it is possible to use the same cata$\log$ for analyzing a service management process in different scenarios. Obviously, this reduces the required effort immensely.

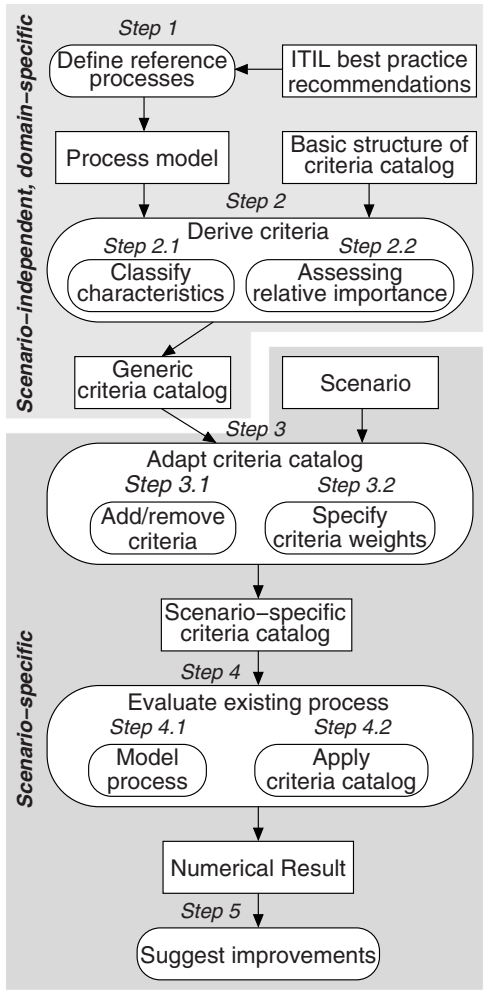

Fig. 1. Analysis Methodology

For developing the criteria catalog, we first define a domain-specific reference process which represents the optimal implementation of the service management process that we want to analyze (step 1). Fortunately, there exists a widely accepted framework detailing the best practice in realizing service management processes, namely the ITIL documents. Thus, we are developing a process model of the "ideal" reference process by using the ITIL recommendations concerning the process of interest. For the purpose of deriving the actual criteria (step 2), the relevant process characteristics are identified on the basis of the created best practice process model. This step is explained in more detail in Section 4. In brief, process elements (e.g. activities) are identified and their relevant characteristics are distilled and classified (step 2.1). Afterwards, every identified process characteristic of the process element, is examined on requirements that have to be fulfilled for optimal process quality. This leads to a specification of at least one criterion in combination with a discrete range of possible attributes which represent a rating to what extent the corresponding requirement is fulfilled. It has to be stressed that the derived criteria are not measuring the process performance by applying common metrics like "average resolution time". Instead, the criteria specify what elements are needed so that an optimal process implementation is possible. This, of course, could mean that "activity 'measuring average resolution time' exists" is specified as a criterion. Compiling the identified criteria, according to their relative importance (step 2.2), into the catalog's basic structure (see Section 3.2 for more details) yields a domain-specific, but reusable (regarding its application in different scenarios) criteria catalog.

In order to apply the criteria catalog to a concrete service management process for analysis purposes, we first adapt the generic criteria catalog according to scenariospecific circumstances (step 3). This usually means, that new criteria are added or existing ones removed (step 3.1) and the weights of the top-level criteria are determined within their given ranges (step 3.2). It needs to be stressed that step 3 is optional and thus, in many cases the generic criteria catalog is adopted without any changes. For the actual process analysis, one first needs a modeled representation of the given pro- 
cess (step 4.1). Afterwards, aspects of interest regarding the process implementation are rated according to the (adapted) criteria catalog (step 4.2). Finally, an overall score is computed on the basis of the single ratings, which represents the measured quality of the examined management process. On the basis of the evaluation catalog, less effective aspects of the process implementation can be located easily (identifiable by a rating lower than average) and, with the reference process in mind, improvements can be suggested immediately (step 5).

\subsection{Main Characteristics of a Criteria Catalog}

A criteria catalog basically consists of a structured representation of the gathered criteria, a range of attributes for each criterion and a measurement methodology to calculate the overall score. During our requirements analysis, regarding the most important characteristics of a criteria catalog, we have identified that a criteria catalog has to be precise, easy to apply and also adaptable. In particular we have to emphasize the importance of the latter requirement, i.e. adaptability, which is motivated by two observations: first, as knowledge about what constitutes an optimal process implementation grows with time and consequently reveals either new criteria or changes in existing ones, we need a criteria catalog which is adaptable without the need to restructure or even to respecify the whole criteria catalog. Secondly, while it is the stated goal to develop a generic, scenario-independent catalog, not all criteria from a catalog are applicable to every scenario. It is obvious that there exists a small set of criteria for analyzing process elements which are mandatory for, e.g., large companies but are of minor concern for smaller companies and vice versa. Consequently, it should be possible, to add and/or remove criteria and still be able to produce comparable scores. In the following the design decisions made to meet these mentioned requirements are presented.

Structure of criteria catalog. The catalog's structure in its most general form is a directed, cycle-free graph of criteria, where a criterion is a node and edges represent (weighted) associations between criteria. We distinguish between a leaf criterion, which is directly measurable and an inner node criterion, for which the score is computed on basis of the associated "lower" criteria. To avoid too complex dependencies, in most cases the catalog is structured like a tree, where every inner node criterion is only dependent on criteria on the directly neighbored (lower) level. However, the general structure is explicitly not limited to tree-like graphs, as there are reasonable use cases where one "child" criterion is associated to more than one "parent" criterion. In any case, there exists exactly one root criterion expressing the overall achieved score of the measured process.

Range of attributes. Every (and solely a) leaf criterion is associated with a range of attributes, which represent the possible ways to meet the criterion. These attributes are distilled from analyzing the reference process, i.e. in our case a process model generated on basis of the ITIL recommendations. The way how to define attributes on the basis of a reference process is illustrated in Section 4.

Measurement methodology. Every attribute of a leaf criterion represents a qualitative level. Since the analysis must be quantifiable to allow comparisons, we need a suitable translation of qualitative levels into numerical values. The number of levels needs to be large enough to be expressive, but small enough to keep the matching of manifestations to scores simple. Therefore, we suggest a rating scale from 0 to 3 points with a 
corresponding attribute-independent meaning about the level of achievement (see Table 1). The actual attributes of a criterion are mapped to the manifestations mentioned in Table 1. This means that for every rating level an criterion-individual text appears.

Table 1. Rating scale

\begin{tabular}{l|c|c} 
Manifestation & Rating & Score s \\
\hline \hline Solution fully meets the criterion & succeeds & 3 \\
Solution roughly meets the criterion & acceptable & 2 \\
Solution only partially meets the criterion & poor & 1 \\
Solution fails to meet the criterion & fails & 0
\end{tabular}

An example for such a mapping is given in the next section in Table 4.

To express differences in the significance for computing the score of an inner node "parent" criterion on basis of associated "child" criteria, we additionally specify a weighting schema (see Table 2). The more important a single criterion in comparison to criteria associated to the same "parent" criterion is, the higher its weight is.

Table 2. Weighting scheme

\begin{tabular}{l|c} 
Description & Weight $\mathrm{w}$ \\
\hline \hline criterion is of primary importance & 4 \\
criterion is of major importance & 2 \\
criterion is of minor importance & 1
\end{tabular}

As aforementioned, only leaf nodes are considered as directly measurable, whereas the score of inner node criteria is computed on basis of the associated criteria. The formula that computes the score has to deliver meaningful and easily interpretable results. Adaptations regarding the number of criteria, i.e., adding and/or removing a single criterion, should not have any consequences regarding the comparability of computed scores. Therefore, we suggest that scores for an inner node criterion is computed as the weighted average of the scores achieved by its children. Overall, the score of an inner node criterion $P$ associated to $n$ child criteria is calculated using the following formula:

$s_{P}=\frac{\sum_{i=1}^{n} s_{i} w_{(i \rightarrow P)}}{\sum_{i=1}^{n} w_{(i \rightarrow P)}}$, with $i \rightarrow P$ representing a child-/parent-node-association between criterion $i$ and criterion $P$, respectively. This way it is possible to calculate the score of the root criterion, which corresponds to the overall achieved analysis result, by recursively applying the given formula. The given formula ensures that the computed scores of the inner nodes always remain in the ranges of the given rating scale. This way the resulting scores are consistently interpretable using the rating scale in Table 1.

Benefits. In our opinion, the most relevant advantage of deriving and arranging criteria in the proposed way is that a criteria catalog has to be developed just one time, but can then be applied many times. Especially, as the criteria catalog is adaptable by adding/removing criteria as well as by changing weights, it can serve as a powerful measurement instrument for analyzing management processes in a wide variety of scenarios. 


\subsection{Catalog's Basic Structure for Analyzing Service Management Processes}

Up to this point, the general structure of a criteria catalog has been specified. For the purpose of using the criteria catalog as a tool for analyzing service management processes, we define a process' quality by its major characteristics, i.e. effectiveness, effciency and manageability [2]. Effectiveness determines, whether the process produces the desired output. In contrast to this, efficienc y specifies the amount of resources used and needed to achieve the output. Additionally, manageability defines the adaptability of a process.

By analyzing these three major quality characteristics, we succeeded in identifying six generic analysis criteria. Thus, these criteria form the top level inner node criteria of a corresponding catalog, being positioned right under the root criterion (see Table 3).

Table 3. Basic criteria

\begin{tabular}{|c|c|c|c|}
\hline $\begin{array}{l}\text { Top level inner } \\
\text { node criterion }\end{array}$ & $\begin{array}{r}\text { Inf } \\
\text { effectivenes }\end{array}$ & $\begin{array}{l}\text { fluences } p r \\
\text { slefficiency }\end{array}$ & $\begin{array}{l}\text { rocess' } \\
y \mid \text { manageability }\end{array}$ \\
\hline (1) Effectiveness of activities & $x$ & $x$ & \\
\hline 2 Process integration & $x$ & $x$ & \\
\hline (3) Alignment & & $x$ & $\times$ \\
\hline (4) Automation & & $x$ & \\
\hline (5) Activity ordering & & $x$ & \\
\hline 6 Process documentation & & & $x$ \\
\hline
\end{tabular}

The effectiveness of the complete process is of course determined by the effectiveness of the process' single activities $(\mathbf{(})$. We identified that the level of process integration (2) is an additional relevant factor, since lost, misdirected or superfluous activity output does not contribute to the overall process' effectiveness. As the process' efficenc y is negatively affected when a process crosses too many organizational boundaries, we specified the alignment (3) of process boundaries as an additional top level criterion. Alignment measures, whether all specified roles of the process are assigned to the appropriated persons, tools or business functions [2]. Furthermore, alignment also influences the process manageability, if, e.g., parts of the process lie outside of the sphere-of-influence of the process owner. The efficienc y is obviously also impacted by the level of automation (4), i.e. the efficient use of IT-tools, etc., and the optimality of activity ordering (-), which determines, if, e.g., activities could be further parallelized. Finally, a sufficient process documentation $(\boldsymbol{6})$ is indispensable for the manageability of the process. In the following section, a criteria catalog is developed for the Incident Management process using the introduced methodology and the presented basic structure of the catalog.

\section{Application: Incident Management}

This Section presents an overview of the reference Incident Management process put together from the ITIL guidelines (4.1), followed by a description of how analysis of the characteristics of this process permitted the derivation of a criteria catalog for evaluating IT Incident Management (4.2). Section 4.3 recounts the experiences in applying this catalog in a cooperation with the User Service Center (USC) of the BMW Group. 


\subsection{Process Model According to ITIL}

As mentioned above, the ITIL does not use a structured notation nor provides exact models of the service management processes it defines. However, for our purpose of using a "virtual" best practices process to compare existing processes against, we needed a reference process derived from the ITIL guidelines, as well as its complete and formal description.

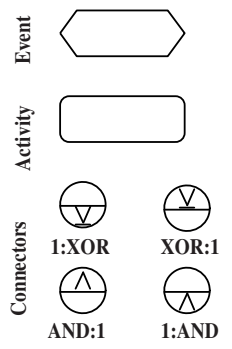

Fig. 2. Basic EPC Elements

Event-driven Process Chains (EPCs) are a semi-formal graphical modeling language for creating workflow graphs [9]. We chose to use EPCs because, due to their use in some of the leading tools for Business Process Reengineering (e.g. ARIS) and Enterprise Resource Planing (e.g. SAP R/3), they have become a widespread method for modeling business processes. Those basic node elements of EPCs that are used in this paper, are depicted in Fig. 2.

In an EPC, a process is modeled by breaking it up into a chain of alternating events and activities. Every EPC has exactly one starting and at least one final event. Activities are triggered by events and are themselves producing events. Note that, in order to have a consistent nomenclature, the term "function", usually used in EPCs, has been replaced with "activity" here.

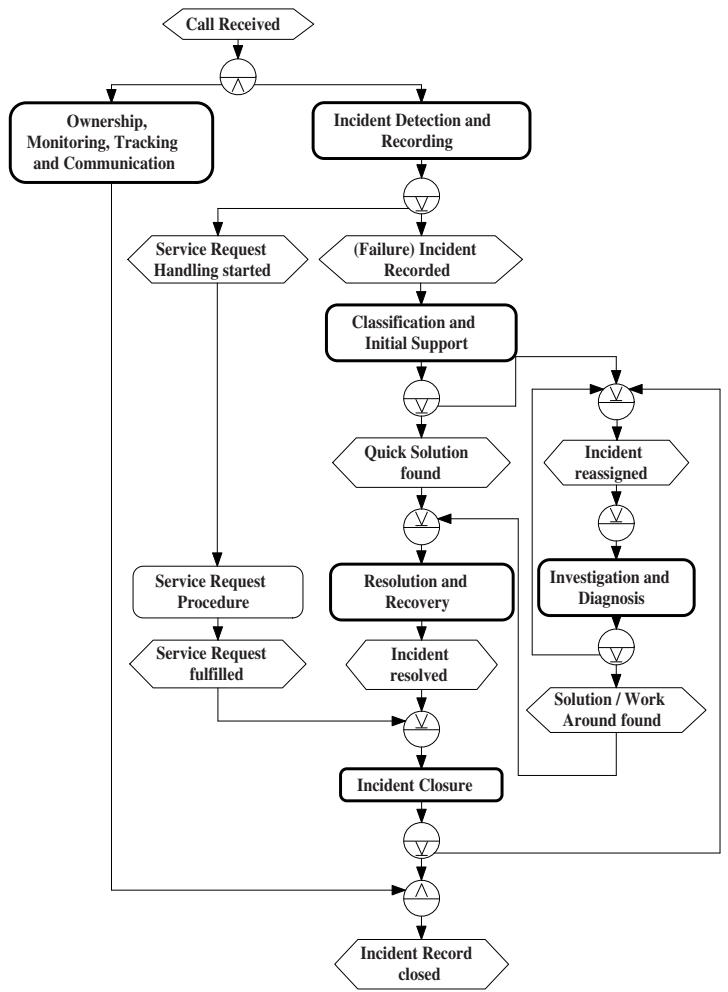

Fig. 3. EPC of the ITIL Incident Management Process
We analyzed the guidelines for the ITIL process Incident Management and used them to build the model of an "exemplary" incident management process (step 1 of the methodology in Section 3.1). The main concern of Incident Management, as defined by ITIL, is restoring the normal service operation after an event (incident) occurs, which causes or may cause a reduction in the quality of that service [8]. A top-level view of our model of the ITIL Incident Management process is displayed in Fig. 3.

The process itself is divided into six sub-processes (activities): Ownership, Monitoring, Tracking and Communication; Incident Detection and Recording; Classification and Initial Support; Service Request Procedure (optional); Investigation and Diagnosis; Resolution and 
Recovery and Incident Closure. While Ownership, Monitoring, Tracking and Communication is a control process, the other activities are concerned with the handling of an incident during the different phases of its life-cycle. With the exception of Service Request Procedure, for which no guidelines exist, each of these activities is a subprocess (or process phase) of our reference process. Accordingly, we created separate EPCmodels for each of them. We also developed a simple model of the information flow among the sub-processes of Incident Management, and between them and other ITSM core processes (not depicted here).

\subsection{Deriving a Generic Criteria Catalog for Incident Management}

This reference process was examined, considering the influence of different process elements on the top-level criteria presented in Section 3.3. As the next step followed the stating of criteria (step 2.1 of the methodology), representing process characteristics derived from the ITIL guidelines or extracted from ITIL self-assessment questionnaires [7]. A (slightly simplified) example of a leaf criterion is depicted in Table 4. We subsequently assigned these leaf criteria to the appropriate top-level criteria described in Section 3.2. Process documentation and activity ordering were each assigned only one single leaf criterion, which was therefore integrated into the respective top-level criterion. Subsequently the weightings were assigned.

Table 4. Criterion: Assignment of Incident Priority

\begin{tabular}{|l|c|c|}
\hline Manifestation & Rating & Score \\
\hline \hline Priority assignment based on (at least) estimated impact and urgency & succeeds & 3 \\
\hline Priority assignment based on (at least) estimated impact & acceptable & 2 \\
\hline Priority assignment based on (at least) estimated urgency & acceptable & 2 \\
\hline Priority assignment based on other considerations & poor & 1 \\
\hline No method for prioritizing incidents & fails & 0 \\
\hline
\end{tabular}

As already mentioned in Section 3.3, the weighting of the top-level criteria depends on various scenario conditions. For example, the importance of Automation depends on the number of incidents handled, Integration on the number of functions (business units) involved in ITSM. Therefore only the possible weight range was assigned to each of these criteria. The relative weights of their sub-criteria however can be assumed to be largely scenario-independent and were derived by their estimated contribution to the respective top-level criterion. The resulting catalog can be seen in Fig. 4 on the next page.

\subsection{Applying the Criteria Catalog at the USC (BMW Group)}

We used this generic criteria catalog for evaluating the performance of the User Service Center (USC) of the BMW Group. The USC controls and coordinates the IT Support efforts for all employees and IT service customers (international distribution centers, dealers, suppliers etc.) of the BMW Group. It runs a 24/7 virtual service desk.

After consultations with our project partner, we adapted the catalog by creating a new root criterion for the overall evaluation of all USC efforts. To this, the generic catalog for Incident Management, as well as two additional criteria for the evaluation of Quality Assurance and Reporting \& Relationship Management were assigned as sub 


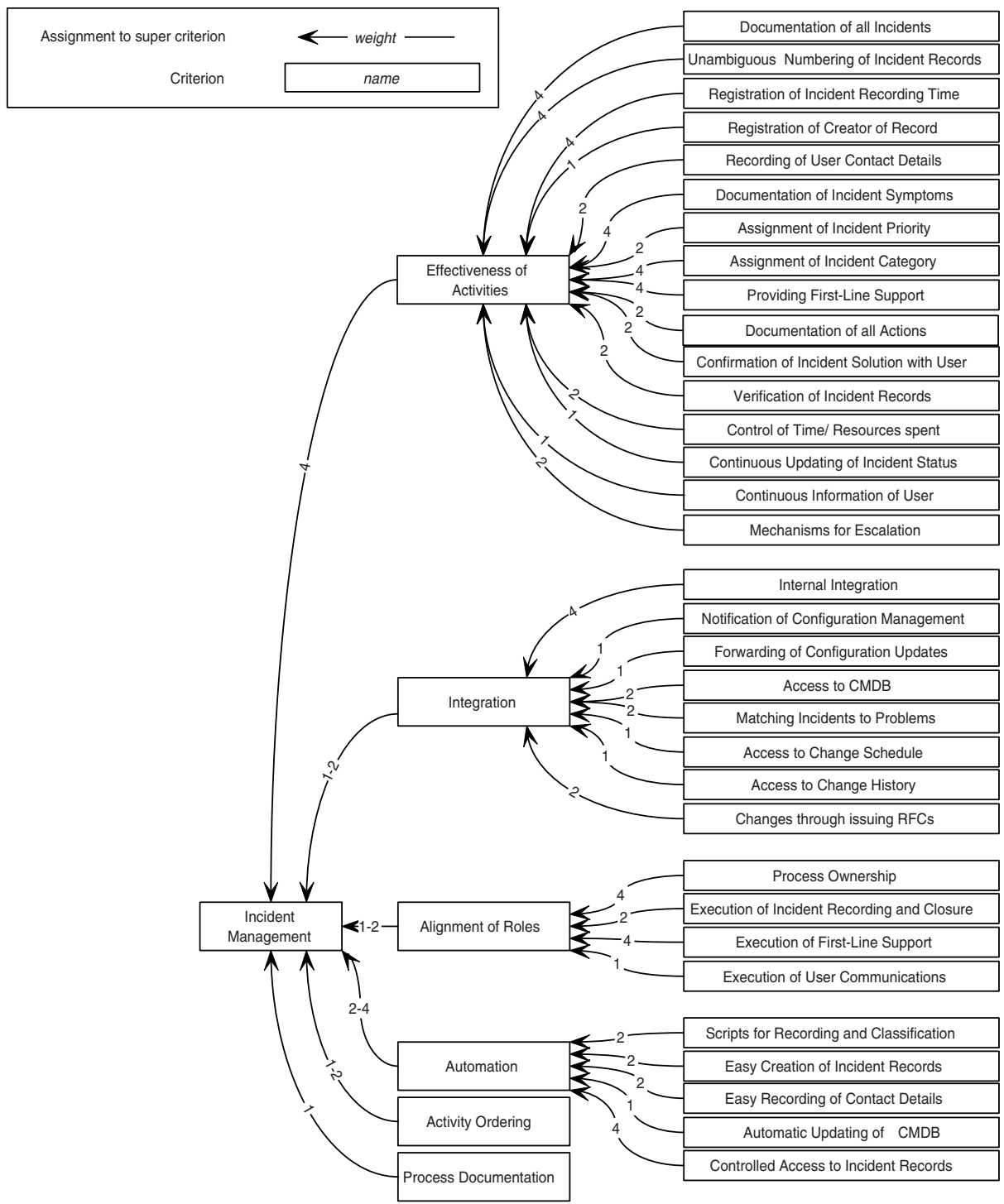

Fig. 4. Generic catalog for Incident Management Processes

criteria (step 3.1 of the methodology). The adaption to the scenario was completed by assigning weights to these new criteria and ascertaining the final weights of the sub criteria of Incident Management (step 3.2 of the methodology). The final catalog is depicted in Fig. 5 on the facing page. For the overall rating of the user service at the BMW Group we weighted the Incident Management Process as "of primary importance", and Quality Assurance as "of major importance". Reporting \& Relationship Management was deemed "of minor importance" relative to the other two criteria. 
As described in Section 3, for applying the Incident Management catalog, the corresponding process at the BMW Group had to be modeled (step 4.1 of the methodology). In this particular case, we were able to reuse an up-to-date model of the User Support processes already developed at the BMW Group with only minor modifications.

At first glance, Incident Handling at the USC and our reference process looked quite different. Further analysis quickly revealed though, that the differences lay mainly in dissimilar naming of equivalent process elements and the different structuring of activities into sub-processes. Despite these apparent discrepancies, the necessary mapping of ac-

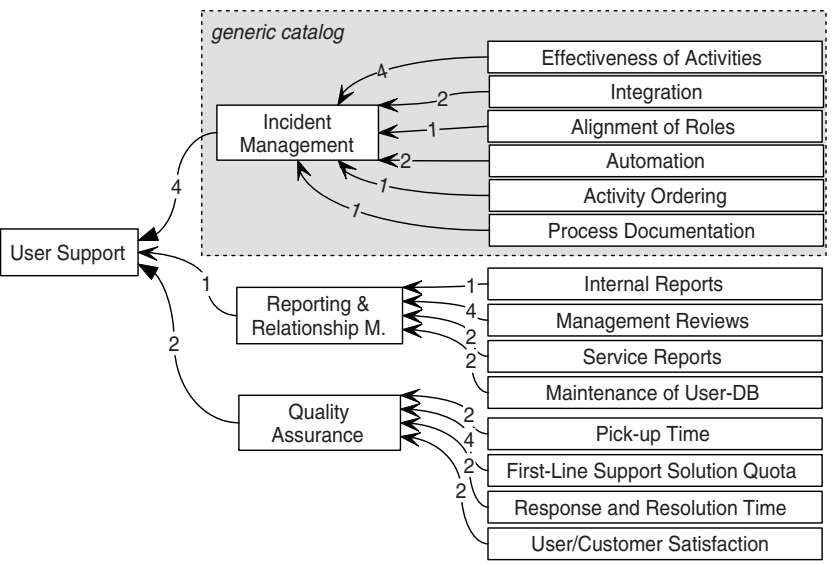

Fig. 5. Specific catalog for evaluating User Support at the USC tivities and events from the USC process to their counterparts in our reference process, and therewith to the corresponding criteria, was achieved without much difficulty. By examining both models, inspecting the implementation of the activities at the USC and analyzing the Service Desk Tool used there, we were able to answer the questions in the catalog and award the corresponding points to the basic criteria in a relatively short time (step 4.2 of the methodology). Once the evaluation of all basic criteria had been completed, the values for the inner node criteria and the root criterion were calculated.

For each of the inner node criteria, we plotted the values assigned to its respective subcriteria into a net-diagram. This way, it was possible to identify particular strengths and weaknesses of the processes as well as especially worthwhile improvement opportunities (step 5 of the methodology). For example, the diagram depicted in Fig. 6 showed a weakness in the

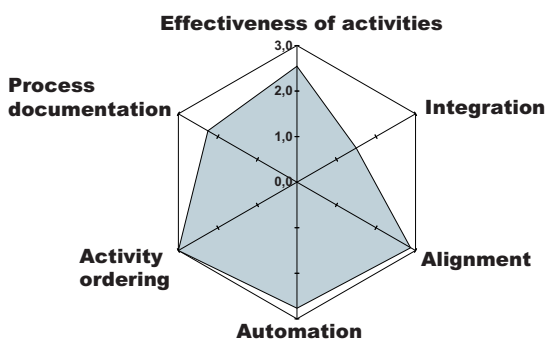

Fig. 6. Results (example) Integration of the Incident Management process at the USC. Further analysis (by examining the diagram of the Integration evaluation) quickly revealed that, while internal integration was excellent, many means for integrating Incident Management with other ITSM processes had not yet been implemented. The results of this analysis are being used in a restructuring project at the BMW Group, with the goal of achieving a better service quality through usage of the ITIL framework. 


\section{Conclusion and Further Work}

The presented work is a result of a research cooperation between the BMW Group and the Munich Network Management Team. Its primary goal was to identify quality decreasing parts of the implementation of service management processes, especially the Incident Management, of the BMW Group's User Service Center, and to suggest improvements. Since our results should support decisions regarding the reorganization of the process implementation, they had to be based on an objective analysis. For this purpose, we performed a systematic analysis and evaluation of the existing processes. These were evaluated on a fix ed set of decision criteria derived on basis of the ITIL framework. The identified criteria were combined to a criteria catalog, which in combination with a specified measurement methodology, provided a comprehensive instrument for analyzing the USC processes.

Besides the development of a specific criteria catalog for evaluating Incident Management processes, a methodology is provided for both creating a criteria catalog and carrying out the analysis. We consider the presented methodology for creating and adapting criteria catalogs our most valuable contribution. Due to their adaptability, catalogs can easily be modified to reflect changing demands on the process implementation or gains in the knowledge about the "best practice". While being comprehensive and competitive in comparison to previously developed analysis methods, the presented methodology remains uncomplicated and easy-to-use.

Our current work is concentrating on considering complementary factors like economic aspects of change activities needed for improvement like acquisition costs, training of management staff, etc. This certainly could lead to a different analysis result. Furthermore, we are examining use cases which would justify the more complex computation of scores in a non tree-like catalog structure.

\section{References}

[1] M. Garschhammer, R. Hauck, B. Kempter, I. Radisic, H. Roelle, and H. Schmidt. The MNM Service Model - Refined Views on Generic Service Management. Journal of Communications and Networks, 3(4):297-306, December 2001.

[2] M. Hammer and J. Champy. Reengineering the Corporation - A Manifesto for Business Revolution. HarperCollins, May 1993.

[3] H.-G. Hegering, S. Abeck, and B. Neumair. Integrated Management of Networked Systems Concepts, Architectures and their Operational Application. Morgan Kaufmann Publishers, ISBN 1-55860-571-1, 1999. $651 \mathrm{p}$.

[4] R. Hiebeler, C. Ketteman, and T. B. Kelly. Best Practices - Building Your Business With Customer-Focused Solutions. Simon and Schuster, 1998.

[5] W. J. Kettinger, J.T.C. Teng, and S. Guha. Business process change: A study of methodologies, techniques, and tools. MIS Quarterly, 21(1), Mar 1997.

[6] J. Kraft. The Department of the Navy Benchmarking Handbook - A Systems View. Department of the Navy — Total Quality Leadership Office, 1997.

[7] Office of Government Commerce (OGC). Service support self assessments.http: / / www . ogc.gov.uk/itil/online_ordering/online_ordering.htm, 2002.

[8] Office of Government Commerce (OGC), editor. Service Support. IT Infrastructure Library (ITIL). The Stationary Office, Norwich, UK, 2000.

[9] A. Scheer. Business Process Engineering - Reference Models for Industrial Companies. Springer Verlag, 2nd edition, 1994. 